\title{
A Case of Chronic Infectious Arthritis of the Temporomandibular Joint Associated with Osteomyelitis without Malocclusion
}

\author{
Shinji Kito ${ }^{1}$, Souichi Hirashima ${ }^{2}$, Izumi Yoshioka ${ }^{3}$, Manabu Habu ${ }^{3}$, Masaaki Kodama ${ }^{3}$, Shinya Ko- \\ kuryo ${ }^{3}$, Masafumi Oda ${ }^{1}$, Tatsurou Tanaka ${ }^{1}$, Nao Wakasugi-Sato ${ }^{1}$, Shinobu Matsumoto-Takeda ${ }^{1}$, \\ Yuji Seta ${ }^{4}$, Kazuhiro Tominaga ${ }^{3}$, and Yasuhiro Morimoto
}

\author{
Departments of ${ }^{1}$ Oral Diagnostic Science, ${ }^{3}$ Oral and Maxillofacial Surgery, and ${ }^{4}$ Bioscience, Kyushu Dental College, \\ Kitakyushu, Japan, ${ }^{2}$ Department of Dentistry and Oral Surgery, University Hospital of Occupational and Environmental \\ Health, Kitakyushu, Japan
}

\begin{abstract}
Infectious arthritis of the temporomandibular joint (TMJ) is rare, and previous reports have identified malocclusion resulting from condylar deformity and displacement of the condyle as one of the clinical characteristics of the disease. Here we report the case of a 33-year-old man with chronic infectious arthritis of the TMJ without malocclusion associated with osteomyelitis of the right mandible. Based on radiological findings of more prominent inflammation at the TMJ than in other regions and on the observed efficacy of antibiotic administration, we made a diagnosis of suppurative arthritis of the TMJ. Based on our empirical experience, including the present case, we speculate that refusal to cooperate with medical care may be a factor in the development of infectious arthritis of the TMJ.
\end{abstract}

Keywords: Infectious arthritis, temporomandibular joint, malocclusion.

\section{CASE REPORT}

A 33-year-old man presented to our dental hospital with the chief complaint of pain and swelling in the right mandible, including increasing difficulty opening his mouth, which had persisted for 3 years. The patient reported that he had experienced no bacterial infection in the orofacial area and had no oral habits that could be expected to affect the tongue. Clinical findings showed that the right mandible was prominently swollen and asymmetric due to the long-term swelling over the past 3 years (Fig. 1). However, the patient did not remember any episodes of fever during the previous 3 years, and he did not have fever at initial presentation. Blood test results were as follows: hematocrit, $35.0 \%$ (slightly low); hemoglobin, $10.8 \mathrm{~g} / 100 \mathrm{ml}$ (slightly low); white blood cell count, $6620 / \mathrm{mm}^{3}$ (normal); CRP, 1.0 (slightly high); and erythrocyte sedimentation rate, 87 (high). Rheumatologic disease was not indicated by the blood test. The patient had not experienced traumatic damage to the mandible.

Panoramic radiographs and X-ray computed tomography (CT) images clearly showed a prominent deformity with bone destruction of the right mandible, including the right condyle (Figs. 2 and 3). In addition, CT images showed swelling and an unclear margin of soft tissue, as well as cloudy fat tissue around the mandible at the center of the TMJ (Fig. 3). Magnetic resonance imaging (MRI) showed inflammatory findings in the mandible, such as MR signal alteration, mandibular and condylar deformation, and bone destructions without mass lesions (Fig. 4). MRI also revealed

\footnotetext{
*Address correspondence to this author at the Division of Diagnostic Radiology, Department of Oral Diagnostic Science, Kyushu Dental College, 2-61 Manazuru, Kokurakita-ku, Kitakyushu 803-8580, Japan; Tel: 81-93-2853094; Fax: 81-93-285-3094; E-mail: rad-mori@kyu-dent.ac.jp
}

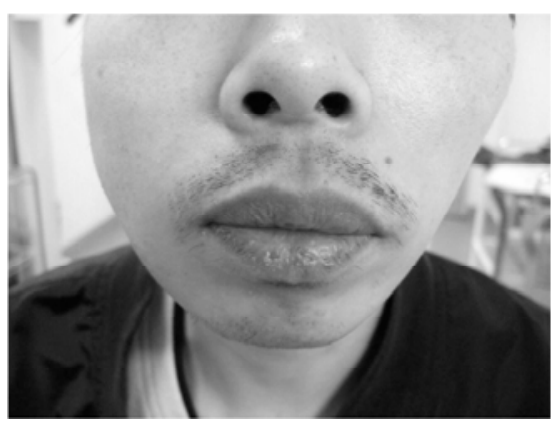

Fig. (1). Photograph of facial appearance of a 33-year-old man shows that the right cheek is prominently swollen and asymmetric.

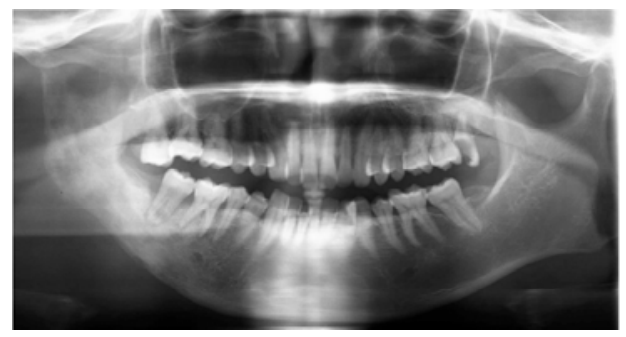

Fig. (2). Imaging findings of the right mandible and temporomandibular joint. Panoramic radiograph reveals deformity of the right mandible and condyle with destruction of cortical and spongy bone. Bilateral mandibular wisdom teeth were defective, and bilateral maxillary wisdom teeth projected into the occlusal plane.

swelling and an unclear margin of soft tissue, as well as high-signal intensity changes around the mandible, including the TMJ (Fig. 4). The inflammatory changes in soft tissues at the TMJ were more prominent than those in the mandible (Fig. 4). However, malocclusion resulting from condylar deformity and anterior displacement of the condyle, a typical finding in chronic arthritis of the TMJ, was not found on 

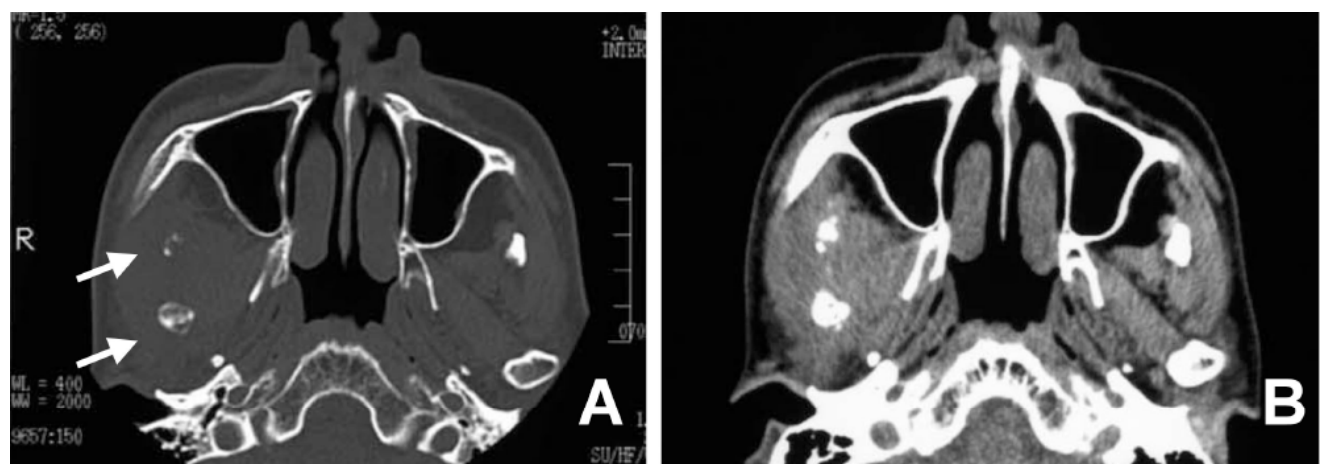

Fig. (3). A. Bone target CT image reveals deformity of the right coronoid process and condyle with destruction of cortical and spongy bone (arrows). B. Soft tissue target CT images show swelling, unclear margin of soft tissue, and cloudy fat tissue around the TMJ.
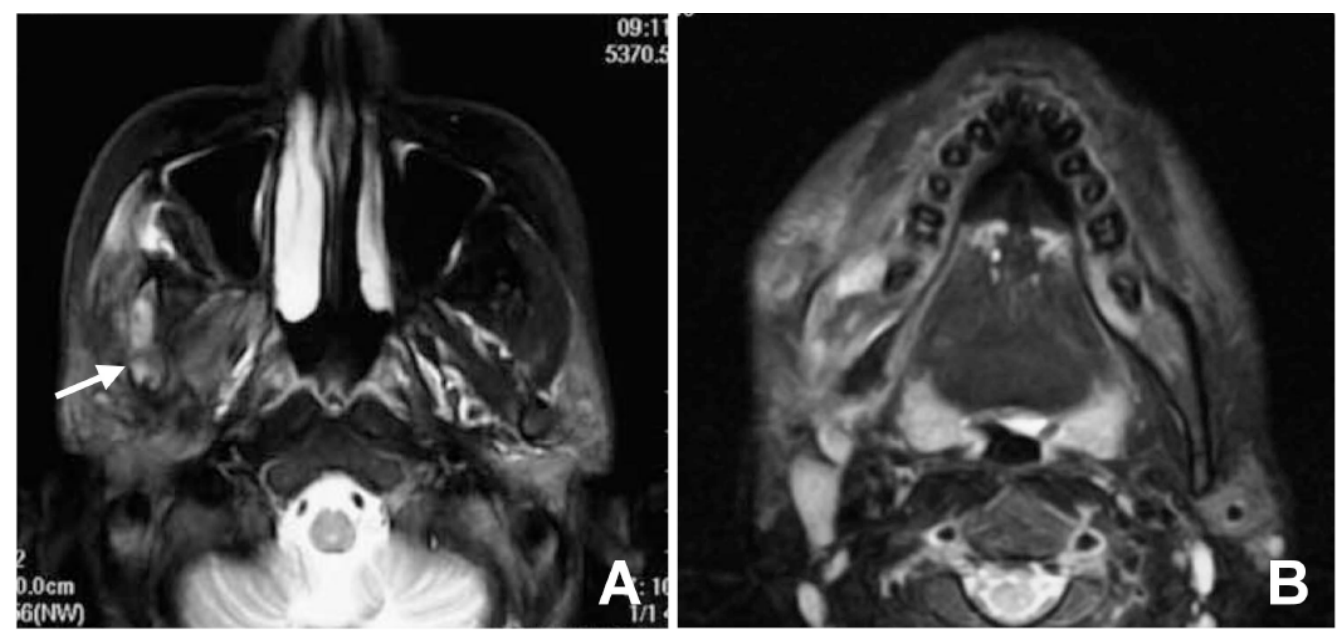

Fig. (4). A. T2-weighted image of the TMJ reveals inflammatory findings in the right TMJ, such as MR signal alteration, mandibular and condylar deformations, and bone destructions without mass lesions (arrow). B. T2-weighted images of the mandible show swelling, unclear margin of soft tissue, and high-signal intensity changes around the mandible, including the TMJ. The inflammatory changes in soft tissues at the TMJ were more prominent than those in the mandible (A, B).

three-dimensional (3D) CT images (Fig. 5) or following oral examination. In the left mandible, no relevant findings were observed on 3D CT of the mandible and occlusions, including the TMJ. In addition, the patient stated that he had no trouble moving the mandible despite the difficulty he experienced opening his mouth.

We clinically diagnosed this case as chronic infectious arthritis of the TMJ associated with osteomyelitis of the right mandible, and immediately administered intravenous cephem antibiotics. After then oral cephem antibiotics was also administrated for 3 days. At a follow-up examination 3 days after starting antibiotics, the patient's pain, swelling, and difficulty in opening the mouth were markedly decreased. Particularly on the extent of opening mouth, the patient recovered from 0.5 of the laid finger to about 2 . Therefore, we continued administration of oral cephem antibiotics for 3 more days. However, the patient has not since returned to our dental hospital. We speculate that the pain and swelling in the TMJ and the patient's difficulty in opening his mouth have since disappeared.

\section{DISCUSSION}

Infectious arthritis of the TMJ has been reported infrequently. In a search of the literature, Sembronio et al. [1] identified approximately 30 reports of infectious arthritis of the TMJ in adults including 6 cases of infectious arthritis of the TMJ in children [2-6]. In previous reports, the clinical manifestations of infectious arthritis have included pain and swelling in the TMJ, difficulty opening the mouth, deviation of the mandible to the opposite side, and malocclusion [119].

Previous reports identify malocclusion as one of the most common clinical manifestations of infectious arthritis of the TMJ; however, malocclusion was not observed in the present case. Despite the lack of malocclusion in the present case, the right mandible and mandibular condyle showed clear deformation equal to or greater than that observed in previously described cases $[1,2,15]$. Thus, the extent of deformity in the present case was sufficient to induce malocclusion. One possible explanation is that the gradual deformity of the TMJ caused by infectious arthritis over the course of 3 years allowed the patient to adapt to his malocclusion. The occlusion of the left side is therefore expected to be normal in the present case despite the anterior displacement of the left mandibular condyle. The present patient had a defect of the bilateral mandibular wisdom teeth, and the bilateral maxillary wisdom teeth projected into the occlusal plane. Malocclusion of the left side might occur in this patient in the fu- 

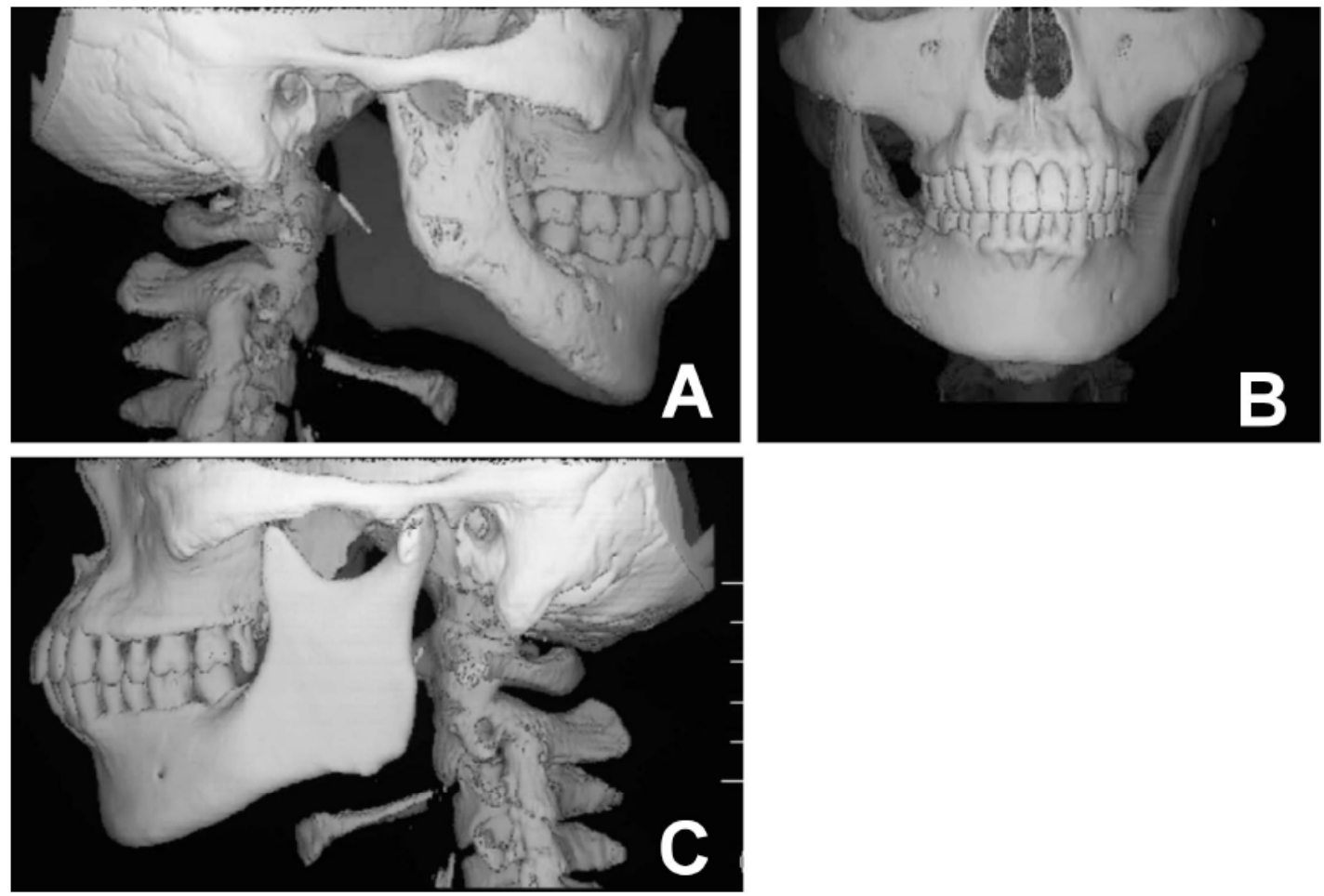

Fig. (5). A. Three-dimensional CT images from the right side view. B. Three-dimensional CT images from the frontal view. C. Threedimensional CT images from the left side view. Three-dimensional CT images reveal deformations and anterior displacement of the right mandible (A, B) and condyle without malocclusion (A, B). No particular findings were noted in the mandible and occlusions, including the TMJ, on 3D CT images of the left side $(\mathbf{C})$.

ture as a result of this condition; however, occlusion was normal at the time of examination except for slight difficulty opening the mouth. This case of infectious arthritis without malocclusion is much rarer than the typically observed form of infectious arthritis of the TMJ, and we must be aware of the presence of infectious arthritis of the TMJ without malocclusion.

The differential diagnosis for infectious arthritis includes fractures, traumatic effusions, cellulites [20], malignant otitis externa [21], gout, pseudogout [22, 23], rheumatologic disease [13], ganglion cysts [24], condylar hypertrophy, and neoplasm [25]. However, we were able to easily diagnose the present patient with infectious arthritis based on the observed efficacy of cephem antibiotics and radiological findings such as panoramic radiographs, CT, and MRI. Using these modalities, we were able to rule out the presence of tumor-like structures in this case. Ipsilateral joint space widening was demonstrated by $\mathrm{CT}$ and MRI. In addition, CT and MRI were useful for the evaluation of both mandibular osteomyelitis and deformity of the TMJ. Certainly, imaging plays an important role in differentiating infectious arthritis of the TMJ from the other diseases mentioned above. The cause of infection is typically either local spread or hematogenous dissemination from a distant site secondary to a systemic process-usually, various head and neck infections. In this case, the infection may have been a result of chronic osteomyelitis; the mandibular deformity, the irregularity in spongy bone in the mandible, and the destruction of the mandibular cortical bone by osteomyelitis. However, inflammatory changes in soft tissues at the TMJ were more prominent than those in the mandible. Therefore, we could not conclude that the inflammation originated solely from mandibular osteomyelitis. Inflammatory changes, including destruction and deformity resulting from mechanical stress, occurred in the TMJ, creating an ideal environment for bacterial infection. After mandibular osteomyelitis or another type of infection, prominent inflammatory changes may have been induced by synergistic effects. The inflammation of the TMJ observed in the present patient was clearly related to bacterial infection: this is why the patient's symptoms, such as difficulty opening the mouth, swelling, and pain in the left TMJ and mandible, promptly resolved after oral and intravenous administration of cephem antibiotics. However, we were unable to identify the causative microorganism. Although Staphylococcus aureus is the most commonly isolated microorganism in patients with infectious arthritis [2], several previous reports [2] have also failed to identify the bacteria responsible for the infection. One of the reasons for this is that adequate examination cannot be performed in patients who, like the present patient, refuse to cooperate in receiving medical treatment. Predisposing factors such as blunt trauma [12, 13], burn wounds [8], iatrogenic causes [14], dental extraction [15], systematic and autoimmune diseases [16, 17], prolonged use of systemic steroids [10], and sexually transmitted diseases $[18,19]$ are commonly identified as playing a role in the development of infectious arthritis of the TMJ. We propose that non-cooperation with treatment should also be considered a predisposing factor, as appropriate medical treatment, including administration of antibiotics, may not be applied in such patients. People in re- 
gions where antibiotics are uncommon or unavailable may also be predisposed to infectious arthritis of the TMJ.

In conclusion, we report a rare case of chronic infectious arthritis of the TMJ in which malocclusion was not observed. As the present patient was not cooperative in receiving medical treatment, we propose that non-cooperation with treatment should be considered a predisposing factor for the development of infectious arthritis of the TMJ, and we must be aware of the presence of infectious arthritis of the TMJ without malocclusion.

\section{REFERENCES}

[1] Sembronio S, Albiero AM, Robiony M, Costa F, Toro C, Politi M. Septic arthritis of the temporomandibular joint successfully treated with arthroscopic lysis and lavage: case report and review of the literature. Oral Surg Oral Med Oral Pathol 2007; 103: e1-6.

[2] Leighty SM, Spach DH, Myall RW, Burns JL. Septic arthritis of the temporomandibular joint: review of the literature and report of two cases in children. Int J Oral Maxillofac Surg 1993; 22: 292-7.

[3] Parmar J. Case Report: septic arthritis of the temporomandibular joint in a neonate. Br J Oral Maxillofac Surg 2008; 46: 505-6.

[4] Amos MJ, Patterson AR, Worrall SF. Septic arthritis of the temporomandibular joint in a 6-year-old child. $\mathrm{Br} \mathrm{J}$ Oral Maxillofac Surg 2008; 46: 242-3.

[5] Regev E, Koplewitz BZ, Nitzan DW, Bar-Ziv J. Ankylosis of the temporomandibular joint as a sequela of septic arthritis and neonatal sepsis. Pediatr Infect Dis J 2003; 22: 99-101.

[6] Viraraghavan R, Kelly L, Podstreleny S, Obeid G. Fever and jaw pain in a five-year-old. TMJ septic arthritis. Pediatr Infect Dis J 2000; 19: 1113, 1115-6.

[7] Goodman WS, Strelzow VV. Infections of the temporomandibular joint. J Otolaryngol 1979; 8: 250-4.

[8] Hilbert L, Peters WJ, Tepperman PS. Temporomandibular joint destruction after burn. Burns 1983; 10: 214-6.

[9] Murakami K, Matsumoto K, lizuka T. Suppurative arthritis of the temporomandibular joint. J Oral Maxillofac Surg 1984; 12: 41-5.

[10] Thomson HG. Septic arthritis of the temporomandibular joint complicating otitis externa. J Laryngol Otol 1989; 103: 319-21.

[11] Trimble LD, Schoenaers JA, Stoelinga PJ. Acute suppurative arthritis of the temporomandibular joint in a patient with rheumatoid arthritis. J Maxillofac Surg 1983; 11: 92-5.
[12] Borenstein DG, Simon GL. Hemophilus influenzae septic arthritis in adults: a report of four cases and a review of the literature. Medicine (Baltimore). 1986; 65: 191-201.

[13] Bounds GA, Hopkins R, Sugar A. Septic arthritis of the temporomandibular joint a problematic diagnosis. Br J Oral Maxillofac Surg 1987; 25: 61-7.

[14] McCain JP, Zabiegalski NA, Levine RL. Joint infection as a complication of temporomandibular joint arthroscopy: a case report. J Oral Maxillofac Surg 1993; 51: 1389-92.

[15] Moses JJ, Lange CR, Arredondo A. Septic arthritis of the temporomandibular joint after the removal of third molars. J Oral Maxillofac Surg 1998; 56: 510-2

[16] Trimble LD, Schoenaers JA, Stoelinga PJ. Acute suppurative arthritis of the temporomandibular joint in a patient with rheumatoid arthritis. J Maxillofac Surg 1983; 11: 92-5.

[17] O'Meara PM, Bartal E. Septic arthritis: process, etiology, treatment outcome: a literature review. Orthopedics 1988; 11: 623-8.

[18] Keat A, Thomas B, Hughes R, Taylor-Robinson D. Chlamydia trachomatis in reactive arthritis. Rheumatol Int 1989; 9: 197-200.

[19] Charles HH, Alan PH, Hervé CG, Pedro FF, Larry MW. Identification of Chlamydia trachomatis in the human temporomandibular joint. J Oral Maxillofac Surg 1999; 57: 683-8.

[20] Hekkenberg RJ, Piedade L, Mock D, Baker G, Freeman JL. Septic arthritis of the temporomandibular joint. Otolaryngol Head Neck Surg 1999; 120: 780-2.

[21] Goldschmidt MJ, Butterfield KJ, Goracy ES, Goldberg MH. Streptococcal infection of the temporomandibular joint of hematogenous origin: a case report and contemporary therapy. J Oral Maxillofac Surg 2002; 60: 1347-53.

[22] Li-Yu J, Schumacher HR Jr, Gratwick G. Invasive tophaceous pseudogout in the temporomandibular joint: misdiagnosis as tumor: case report and review of the literature. J Clin Rheumatol 2000; 6: 272-7.

[23] Nakagawa Y, Ishii H, Shimoda S, Ishibashi K. Pseudogout of the temporomandibular joint: a case report. Int J Oral Maxillofac Surg 1999; 28: 26-8.

[24] Copeland M, Douglas B. Ganglions of the temporomandibular joint: case report and review of literature. Plast Reconstruct Surg 1988; 81: 775-6.

[25] Shapiro BL, Gorlin RJ. Disorders of the temporomandibular joint. In: Gorlin RJ, Goldman HM, Eds. Thomas's oral pathology. $6^{\text {th }}$ ed. St Louis (MO): CV Mosby 1970; pp. 585-8.

(C) Kito et al.; Licensee Bentham Open.

This is an open access article licensed under the terms of the Creative Commons Attribution Non-Commercial License (http://creativecommons.org/licenses/by-nc/3.0/) which permits unrestricted, non-commercial use, distribution and reproduction in any medium, provided the work is properly cited. 